\title{
Mixed-mode GPS deformation monitoring - A cost-effective and accurate alternative?
}

\author{
Volker Janssen, Chris Rizos \\ School of Surveying and Spatial Information Systems, The University of New South Wales, \\ Sydney NSW 2052, Australia, email: v.janssen@student.unsw.edu.au
}

\begin{abstract}
During the past few years a methodology has been developed for processing data collected by GPS networks consisting of a mixed set of single-frequency and dual-frequency receivers. The strategy is to deploy a few permanent GPS stations with dual-frequency, geodetic-quality receivers surrounding an 'inner' deformation monitoring network of low-cost, singlefrequency GPS receivers. The dual-frequency GPS network is used to generate a file of 'corrections', analogous to Wide Area DGPS correction models for the distance dependent biases. These 'corrections' are then applied to the double-differenced phase observations from the inner receivers to enhance baseline accuracy, primarily through empirical modelling of the residual atmospheric biases that otherwise would be neglected. Moreover, epoch-by-epoch baseline solutions are preferred in order to detect deformational signals in (near) real-time. Data from two continuous GPS networks have been used to investigate the performance of this configuration under severe ionospheric conditions and in different geographical regions. In the mid-latitude region the L1 baseline repeatability has clearly been improved by $40-50 \%$, while an improvement of about $20 \%$ has been achieved in the equatorial region. The findings also indicate that the proposed procedure is sensitive to extreme ionospheric conditions, such as those experienced in close proximity to the geomagnetic equator during solar cycle maximum periods.
\end{abstract}

Keywords: Mixed-mode GPS, deformation monitoring, ionospheric corrections.

\section{Introduction}

The Global Positioning System (GPS) can be utilised in a wide range of deformation monitoring applications. The decreasing cost of GPS hardware, together with the increased reliability of the technology, facilitates such demanding applications as the monitoring of active volcanoes, tectonic fault lines, landslides, ground subsidence, bridges, dams, high-rise buildings, etc. GPS measurements can be continuous, automatic, conducted in all weather conditions, and provide three-dimensional positioning results.

Deformation monitoring using GPS is usually carried out by installing and operating a local network of GPS receivers located on and around the deforming body. Most continuous GPS deformation monitoring networks are comparatively costly because they rely entirely on the use of dual-frequency instrumentation (e.g. Ogaja et al., 2001; Owen et al., 2000; Wong et al., 2001). In order to keep the cost of such a deformation monitoring system to a minimum, single-frequency GPS receivers need to be used. On the other hand, some atmospheric biases (mainly the ionospheric delay) cannot be accounted for directly if only one frequency is used, resulting in lower accuracy. It is usually assumed that the differential ionospheric and tropospheric delays between two receivers can be ignored if baseline lengths are kept below $10 \mathrm{~km}$. However, especially during solar sunspot cycle maximum periods, ionospheric disturbances have indeed corrupted L1 baseline measurements over distances less than $10 \mathrm{~km}$, adversely affecting baseline repeatability (Janssen et al., 2001).

It is therefore necessary to combine a singlefrequency deformation monitoring network with a small number of dual-frequency receivers surrounding the deformation zone in order to account for these atmospheric effects. Such a mixed-mode configuration offers considerable flexibility and cost savings for deformation monitoring applications, which require a dense spatial coverage of GPS stations, and where it is not possible, nor appropriate, to establish permanent GPS networks using dual-frequency instrumentation. Hartinger \& Brunner (2000) have used such a mixedmode approach to monitor landslides in the Austrian Alps.

\section{Mixed-mode GPS deformation monitoring}

In a mixed-mode GPS network approach, a fiducial network of dual-frequency receivers surrounding the deformation zone can be used to generate empirical 'correction terms'. These can then be applied to the single-frequency observations of the inner network on the deforming body to account for the ionospheric effects, and hence improve baseline accuracy. Figure 1 shows the ideal configuration of such a mixed-mode GPS network where the triangles denote fiducial stations, while the dots indicate single-frequency sites. The reference stations are to be situated outside the deformation zone but within the local tectonic region in order to avoid unwanted displacements of the external network. 


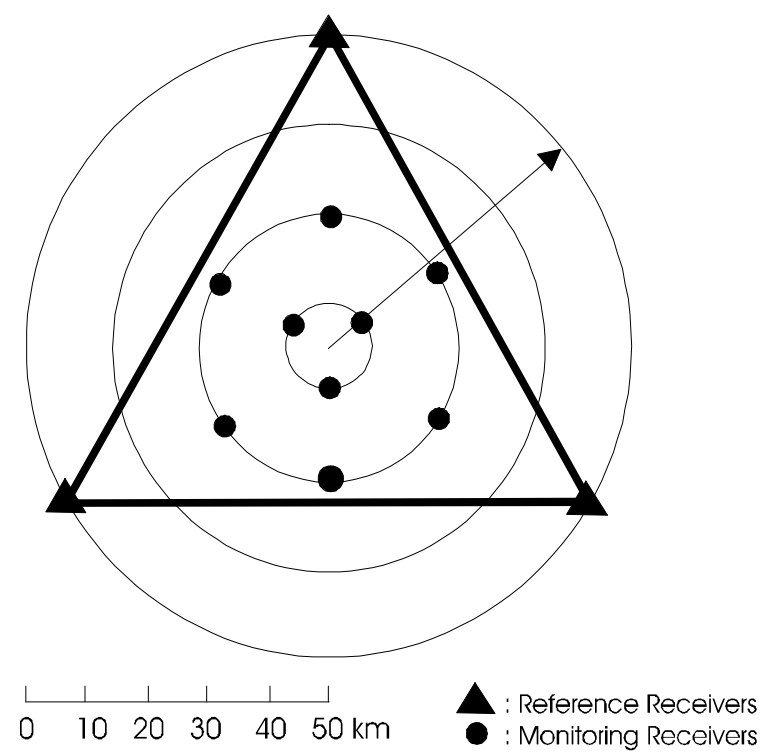

Fig. 1. Ideal network configuration of a mixed-mode GPS deformation monitoring network

A linear combination model, utilising a single ionospheric layer model at a height of $350 \mathrm{~km}$, is used to determine the corrections (Han \& Rizos, 1996; Han, 1997). This approach can account for orbit bias and ionospheric delay, as well as somewhat mitigate tropospheric delay, multipath and measurement noise across the network. Data from the GPS reference station network can thus be used to derive empirical corrections to the double-differenced carrier phase data formed between the stations of the inner network.

If the number of GPS reference stations is assumed to be three, the double-differenced observation equation for a baseline between a reference station and a singlefrequency user receiver located inside the triangle (denoted by u) can be expressed in the form of a linear combination (Han, 1997):

$$
\begin{aligned}
\nabla \Delta \phi_{\mathrm{u}, 3}-\left[\alpha_{1} \cdot \mathrm{V}_{1,3}+\alpha_{2} \cdot \mathrm{V}_{2,3}\right]= \\
\nabla \Delta \rho_{\mathrm{u}, 3}+\lambda \cdot \nabla \Delta \mathrm{N}_{\mathrm{u}, 3}+\varepsilon_{\nabla \Delta \phi}
\end{aligned}
$$

where $\nabla \Delta$ is the double-differencing operator, $\phi$ is the carrier phase observation in units of metres, $\rho$ is the distance between receiver station and satellite, $\mathrm{N}$ is the integer ambiguity for a particular satellite-receiver phase measurement, and $\varepsilon_{\nabla \Delta \phi}$ represents the carrier phase observation noise. The parameters $\alpha$ refer to the position of the user receiver inside the fiducial triangle and can be determined based on the following conditions (Han \& Rizos, 1996; Wu, 1994):

$$
\sum_{i=1}^{3} \alpha_{i}=1, \sum_{i=1}^{3} \alpha_{i} \cdot\left(\vec{X}_{u}-\vec{X}_{i}\right)=0 \text { and } \sum_{i=1}^{3} \alpha_{i}^{2}=\min
$$

where $\overrightarrow{\mathrm{X}}_{\mathrm{u}}$ and $\overrightarrow{\mathrm{X}}_{\mathrm{i}}$ are the user station position vector and reference station position vector respectively, while $\alpha_{i}$ denotes the weight for reference station $i$.
The residual vectors are formed from the doubledifferenced observations between reference stations $1 \&$ 3 and $2 \& 3$ :

$\mathrm{V}_{1,3}=\nabla \Delta \phi_{1,3}-\nabla \Delta \mathrm{N}_{1,3}-\nabla \Delta \rho_{1,3}$
$\mathrm{~V}_{2,3}=\nabla \Delta \phi_{2,3}-\nabla \Delta \mathrm{N}_{2,3}-\nabla \Delta \rho_{2,3}$

The 'correction term' $\left[\alpha_{1} \cdot V_{1,3}+\alpha_{2} \cdot V_{2,3}\right]$ can now be obtained and applied to the user receiver. For a baseline between two GPS stations $\mathrm{j}$ and $\mathrm{k}$ of the inner (singlefrequency) network the linear combination can be expressed as:

$$
\begin{aligned}
\nabla \Delta \phi_{\mathrm{k}, \mathrm{j}}-\left[\alpha_{1}^{\mathrm{k}, \mathrm{j}} \cdot \mathrm{V}_{1,3}+\alpha_{2}^{\mathrm{k}, \mathrm{j}} \cdot \mathrm{V}_{2,3}\right]= & \\
& \nabla \Delta \rho_{\mathrm{k}, \mathrm{j}}+\lambda \cdot \nabla \Delta \mathrm{N}_{\mathrm{k}, \mathrm{j}}+\varepsilon_{\mathrm{k}, \mathrm{j}}
\end{aligned}
$$

where $\alpha_{i}^{k, j}$ is the difference in the $\alpha_{i}$ value for stations $j$ and $\mathrm{k}$, and $\left[\alpha_{1}^{\mathrm{k}, \mathrm{j}} \cdot \mathrm{V}_{1,3}+\alpha_{2}^{\mathrm{k}, \mathrm{j}} \cdot \mathrm{V}_{2,3}\right]$ is the 'correction term' for the inner baseline between these user receivers.

By forming the double-differenced observables between the inner single-frequency receivers, and using the residual vectors generated from the reference stations, the inner stations' coordinates can be determined without the need to use any GPS reference station observations at all. In practice, holding one reference site fixed, the baselines to the other two sites are processed and 'correction terms' are obtained for both baselines. These are then scaled (by means of the parameters $\alpha_{i}$ ) according to the position of the inner stations inside the reference network triangle to generate double-differenced corrections for the inner baselines.

\section{GPS data processing}

A single-frequency version of the Baseline software package developed at UNSW is used to process the inner network incorporating the 'correction terms', which are determined using a modified version of the Bernese software. For deformation monitoring applications multibaseline processing strategies should be used because all baselines are then computed together, taking into account the between-baseline correlations which arise from observing a GPS network simultaneously. For continuous deformation monitoring a near real-time, epoch-by-epoch solution is preferred in order to detect movements over a short period of time.

\section{Case study: Mid-latitude region}

Data from the Southern California Integrated GPS Network (SCIGN) (SCIGN, 2003) were used to investigate the performance of the mixed-mode network configuration in the mid-latitude region. Figure 2 shows the location of the GPS sites, which are all equipped with dual-frequency receivers. The part of the network used in this study consists of an outer network of three 
sites (FXHS, FMTP, QHTP) surrounding an inner network of three sites (CSN1, OAT2, CMP9). The outer sites were used as fiducial GPS reference stations, indicated by triangles in Figure 2, while the inner sites (indicated by circles) simulated single-frequency receiver stations (by ignoring the observations made on L2). The data were collected under solar maximum conditions on three consecutive days from August 8-10, 2000 (DOY 221-223).

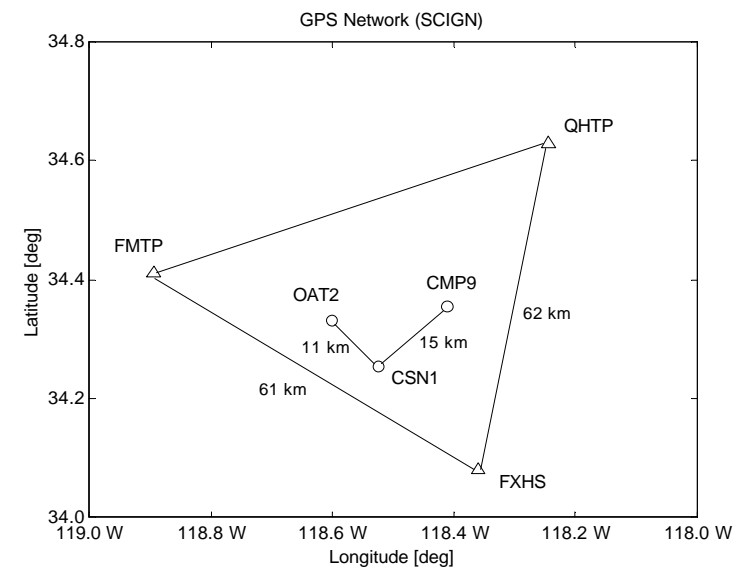

Fig. 2. SCIGN network stations used in this study

The fiducial baselines FXHS-FMTP and FXHS-QHTP are used to model the ionospheric conditions across the network. L1 'correction terms' for the inner baselines CSN1-OAT2 and CSN1-CMP9 can then be determined on a satellite-by-satellite and epoch-by-epoch basis, according to equation (5). As an example, Figure 3 shows the double-differenced corrections obtained over 24 hours for day 221. It can easily be seen that ionospheric activity in mid-latitude regions is mainly a daytime phenomenon. A comparison with directly generated ionospheric delay values for the same baselines (using dual-frequency data) showed that this procedure does indeed compute correct values for the inner baselines.
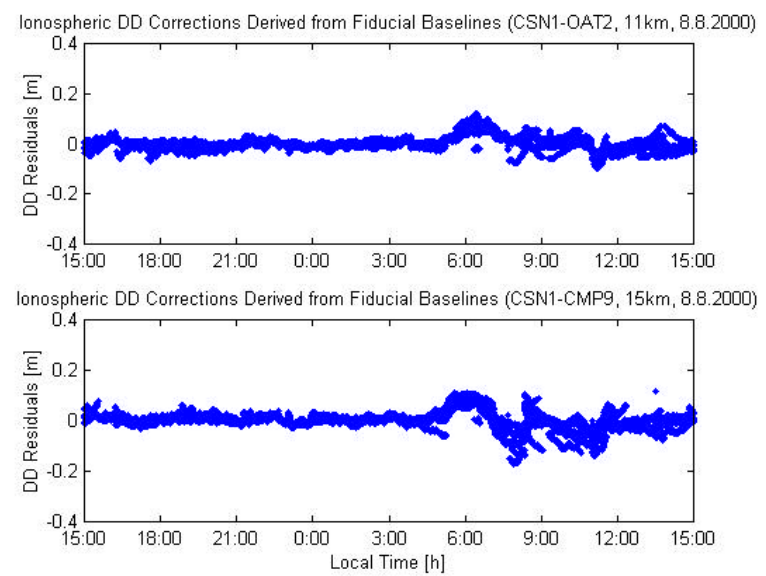

Fig. 3. Double-differenced L1 corrections for the inner baselines (DOY 221)

The Baseline software is then used to process the inner baselines in single-frequency mode, with and without applying the empirically derived corrections. It can readily be assumed that no ground deformation has taken place during the period of observation. Hence, the baseline repeatability gives a good indication of the accuracy that can be achieved with the data processing strategy described in this paper.

Figure 4 shows the results obtained for one inner baseline ignoring ionospheric effects, while Figure 5 shows the results obtained by applying ionospheric corrections on day 221. The graphs show the Easting, Northing and Height components over a 24-hour period, each dot representing a single-epoch solution. The respective standard deviations are also included. In both cases the Saastamoinen model was used to account for the tropospheric bias, as recommended by Mendes (1999).

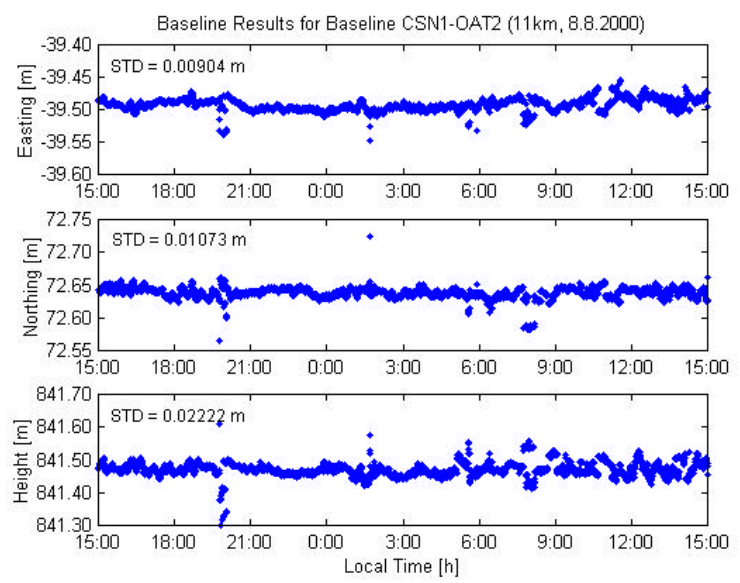

Fig. 4. Results for an inner baseline not using ionospheric corrections (DOY 221)

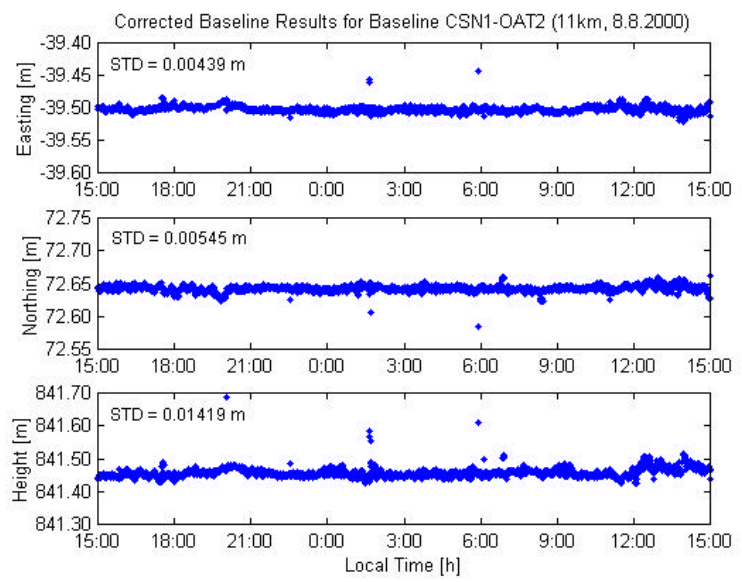

Fig. 5. Results for an inner baseline applying ionospheric corrections (DOY 221)

Similar results were obtained for the second inner baseline and on the following two days. Standard deviations of less than $1 \mathrm{~cm}$ horizontally and $1.5-3 \mathrm{~cm}$ vertically have been achieved for a single-epoch solution by applying the corrections. On average, the standard deviation of the baseline results has been reduced by almost $50 \%$ in the horizontal components and almost $40 \%$ in the vertical component (Table 1). 
Table 1. Average improvement in the STD for both baselines on days 221-223

\begin{tabular}{ccccc}
\hline Baseline & DOY & $\begin{array}{c}\text { East } \\
{[\%]}\end{array}$ & $\begin{array}{c}\text { North } \\
{[\%]}\end{array}$ & $\begin{array}{c}\text { Height } \\
{[\%]}\end{array}$ \\
\hline CSN1- & 221 & 51.4 & 49.2 & 36.1 \\
OAT2 & 222 & 38.1 & 35.8 & 40.7 \\
$(11 \mathrm{~km})$ & 223 & 53.8 & 51.4 & 33.6 \\
\hline CSN1- & 221 & 54.7 & 59.5 & 48.4 \\
CMP9 & 222 & 38.7 & 29.1 & 33.0 \\
$(15 \mathrm{~km})$ & 223 & 56.1 & 55.9 & 34.1 \\
\hline \multicolumn{2}{c}{ Average [\%] } & 49 & 47 & 38
\end{tabular}

\section{Case study: Equatorial region}

In order to investigate the performance of the mixedmode network processing approach in the equatorial region, data from the Hong Kong Active GPS Network (Chen et al., 2001) were used. Figure 6 shows the location of the GPS sites, which are all equipped with dual-frequency receivers. The network consists of an outer network of three sites (HKKY, HKFN, HKSL) surrounding two inner sites (HKKT, HKLT). Due to the absence of a third site within the fiducial triangle, the fiducial site HKKY was used to form the inner (singlefrequency) baselines. Note that HKLT is located just outside the fiducial triangle, which should normally be avoided. However, this does not have any effect on the processing in this case, as HKKY-HKFN and HKKYHKSL are used as fiducial baselines. The data were collected under solar maximum conditions on three consecutive days from October 11-13, 2000 (DOY 285287).

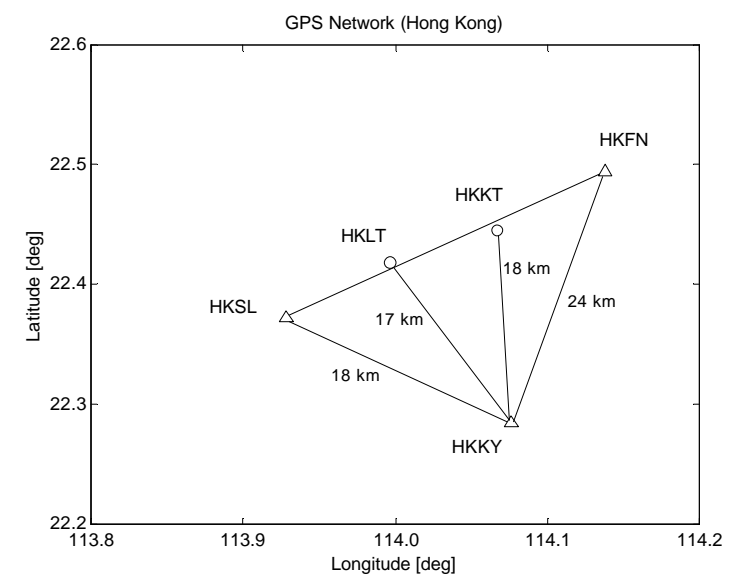

Fig. 6. Hong Kong GPS Active Network stations used in this study

According to equation (1) L1 'correction terms' can be determined for the inner baselines HKKY-HKKT and HKKY-HKLT. As an example, Figure 7 shows the double-differenced corrections obtained for the first day. A comparison with directly generated ionospheric delay values for the same baselines (using dual-frequency data) confirmed the correctness of these values. It is evident that ionospheric activity in the equatorial region is much more severe than in mid-latitudes, reaching values of a few cycles. As expected, most of the activity occurs after local sunset, although there is also a lot of daytime activity. This may be explained by intensified small-scale disturbances in the ionosphere during a period of increased solar activity. Furthermore, it can be identified as the primary diurnal maximum of the equatorial anomaly, also known as the fountain effect (high electron concentration observed on either side of the geomagnetic equator at magnetic latitudes of around $\left.10-20^{\circ}\right)$. The equatorial anomaly has been investigated by, amongst others, Huang \& Cheng (1991) and Skone (2000).

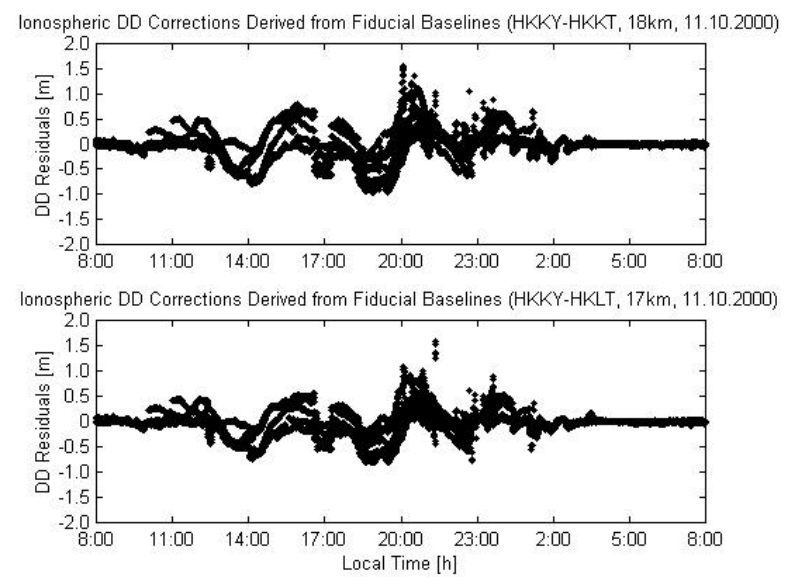

Fig. 7. Double-diff. L1 corrections for the inner baselines (DOY 285)

Again, the baseline processing results for the inner baselines obtained with and without applying the empirically derived corrections were compared. In both cases the Saastamoinen model was used to account for the tropospheric bias. As an example, Figure 8 shows the single-frequency results obtained for one inner baseline ignoring ionospheric effects, while Figure 9 shows the results obtained by applying ionospheric corrections on day 285. The graphs show the Easting, Northing and Height components over a 24-hour period, each dot representing a single-epoch solution. The respective standard deviations are also included. Similar results were obtained for the second inner baseline and on the following two days. Although not obvious from these figures, an improvement in accuracy of around $20 \%$ in all three components has been achieved by applying the corrections (as indicated in Table 2).

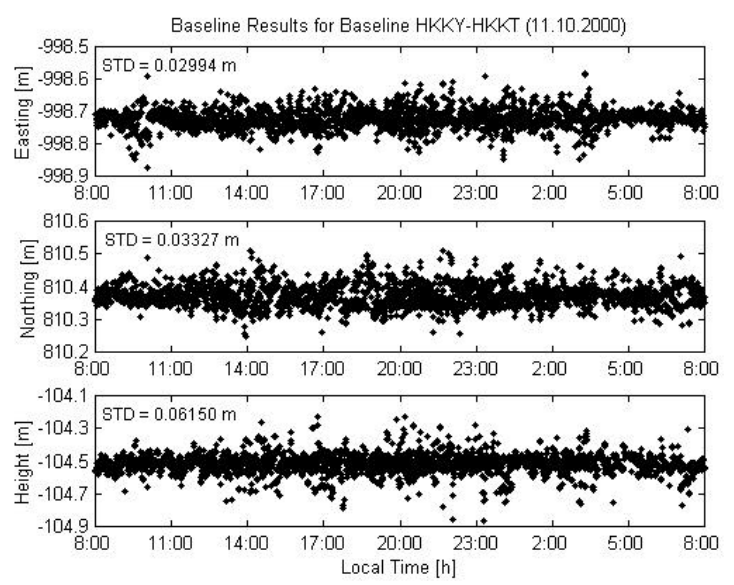

Fig. 8. Results for an inner baseline not using ionospheric corrections (DOY 285) 


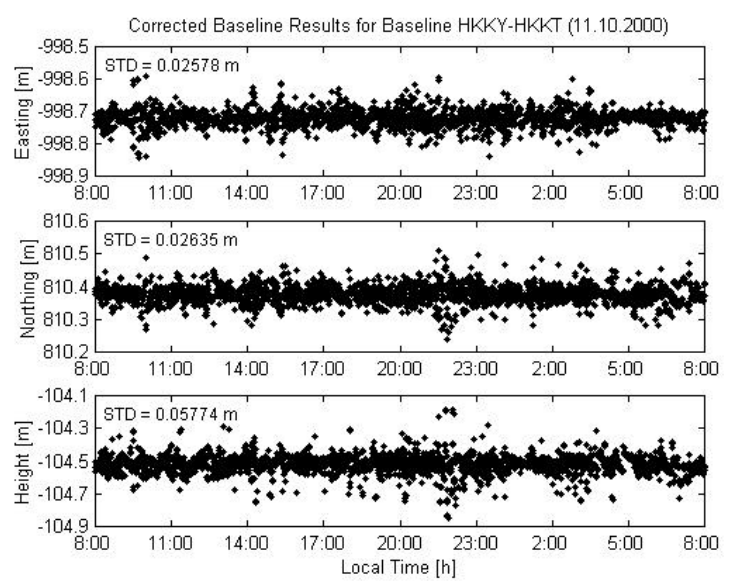

Fig. 9. Results for an inner baseline applying ionospheric corrections (DOY 285)

Table 2. Average improvement in the STD for both baselines on days 285-287

\begin{tabular}{ccccc}
\hline Baseline & DOY & $\begin{array}{c}\text { East } \\
{[\%]}\end{array}$ & $\begin{array}{c}\text { North } \\
{[\%]}\end{array}$ & $\begin{array}{c}\text { Height } \\
{[\%]}\end{array}$ \\
\hline HKKY- & 285 & 13.9 & 20.8 & 6.1 \\
HKKT & 286 & 15.5 & 16.8 & 25.9 \\
$(18 \mathrm{~km})$ & 287 & 23.6 & 24.9 & 26.9 \\
\hline HKKY- & 285 & 15.2 & 19.0 & 5.9 \\
HKLT & 286 & 15.3 & 12.4 & 21.1 \\
$(17 \mathrm{~km})$ & 287 & 25.4 & 26.4 & 26.3 \\
\hline \multicolumn{2}{c}{ Average [\%] } & 18 & 20 & 19 \\
\hline
\end{tabular}

However, in spite of the shorter fiducial baseline lengths compared to the SCIGN network, standard deviations could not be reduced below the few-cm level when corrections were applied. Values of $2.0-3.5 \mathrm{~cm}$ in the horizontal components and $4.5-6.5 \mathrm{~cm}$ in the height component are too high to permit reliable detection of ground deformation at the desired accuracy level. This emphasizes the significant effect of the ionosphere on GPS deformation monitoring networks, especially at low-latitudes in periods of heightened solar activity.

\section{Concluding remarks}

A procedure to process a mixed-mode GPS network for deformation monitoring applications has been described. Single-frequency GPS observations have been improved by generating empirical corrections obtained from a fiducial network of dual-frequency reference stations surrounding the inner single-frequency network. This method accounts for the ionospheric bias that otherwise would have been neglected when using single-frequency instrumentation only. Data from two GPS networks located in different geographical regions have been used to simulate such a network configuration in order to investigate the impact of the proposed processing strategy on baseline results.

In the mid-latitude region the single-frequency baseline repeatability has clearly been improved by applying the empirical 'correction terms'. The standard deviations of the baseline results have been reduced by $40-50 \%$ to less than $1 \mathrm{~cm}$ horizontally and $1.5-3 \mathrm{~cm}$ vertically for a single-epoch baseline solution.
In the equatorial region an improvement of approximately $20 \%$ in all three components has been achieved by applying the corrections. However, the standard deviations could not be reduced below the few$\mathrm{cm}$ level. This indicates that extreme ionospheric conditions, such as those experienced in close proximity to the geomagnetic equator during solar cycle maximum periods, can reduce the efficiency of the proposed method. Nevertheless, the approach of processing a mixed-mode GPS network presented here can be a costeffective and accurate tool for deformation monitoring suitable for a variety of applications. The correction generation algorithm could easily be implemented in a continuous GPS reference station network, distributing ionospheric corrections as a routine product.

Acknowledgements. SCIGN and its sponsors, the W.M. Keck Foundation, NASA, NSF, USGS and SCEC, as well as A/Prof. Peter Morgan from the University of Canberra, are thanked for providing the data used in this study. The first author is supported by an International Postgraduate Research Scholarship (IPRS) and funding from the Australian Research Council (ARC).

\section{References}

Chen W., Hu C., Chen Y., Ding X. and Kwok S.C.W. (2001): Rapid Static and Kinematic Positioning with Hong Kong GPS Active Network. Proc. ION GPS-2001, Salt Lake City, Utah, 11-14 September, 346-352.

Han S. (1997): Carrier Phase-Based Long-Range GPS Kinematic Positioning. PhD Dissertation, UNISURV S-49, School of Geomatic Engineering, The University of New South Wales, Sydney, Australia, 185pp.

Han S. and Rizos C. (1996): GPS Network Design and Error Mitigation for Real-Time Continuous Array Monitoring Systems. Proc. ION GPS-96, Kansas City, Missouri, 17-20 September, 1827-1836.

Hartinger H. and Brunner F.K. (2000): Development of a Monitoring System of Landslide Motions Using GPS. Proc. $9^{\text {th }}$ FIG Int. Symp. on Deformation Measurements, Olsztyn, Poland, Sep 1999, 29-38.

Huang Y.-N. and Cheng K. (1991): Ionospheric Disturbances at the Equatorial Anomaly Crest Region during the March 1989 Magnatic Storms. Journal of Geophysical Research 96(A8): 13953-13965.

Janssen V., Roberts C., Rizos C. and Abidin H.Z. (2001): Experiences with a Mixed-Mode GPS-Based Volcano Monitoring System at Mt. Papandayan, Indonesia. Geomatics Research Australasia 74: 43-58.

Mendes V.B. (1999): Modeling the Neutral-Atmosphere Propagation Delay in Radiometric Space Techniques. PhD Dissertation, Dept. of Geodesy \& Geomatics Eng. Tech. Rept. No. 199, University of New Brunswick, Fredericton, Canada.

Ogaja C., Rizos C., Wang J. and Brownjohn J. (2001): A Dynamic GPS System for On-line Structural Monitoring. Int. Symp. on Kinematic Systems in Geodesy, Geomatics \& Navigation (KIS 2001), Banff, Canada, 5-8 June, 290-297.

Owen S., Segall P., Lisowski M., Miklius A., Murray M., Bevis M. and Foster J. (2000): January 30, 1997 Eruptive Event on Kilauea Volcano, Hawaii, as Monitored by Continuous GPS. Geophysical Research Letters 27(17): 2757-2760.

SCIGN (2003): Southern California Integrated GPS Network (SCIGN) website: http://www.scign.org/.

Skone S.H. (2000): Wide Area Ionosphere Modeling at Low Latitudes - Specifications and Limitations. Proc. ION GPS-2000, Salt Lake City, Utah, 19-22 September, 643-652.

Wong K.-Y., Man K.-L. and Chan W.-Y. (2001): Monitoring Hong Kong's Bridges - Real-Time Kinematic Spans the Gap. GPS World 12(7): 10-18.

Wu J.T. (1994): Weighted Differential GPS Method for Reducing Ephemeris Error. Manuscripta Geodaetica 20: 1-7. 